\title{
THERMAL STRESSES DUE TO INTERNAL HEAT GENERATION IN A
}

\section{THICK CIRCULAR PLATE}

\author{
ANJALI K. SHINDE ${ }^{1}$, ANANT A. NAVLEKAR ${ }^{2} \&$ KIRTIWANT P. GHADLE ${ }^{3}$ \\ ${ }^{I}$ Department of Mathematics, K.R.T.Arts, B.H.Commerce, A.M. Science College, Nasik, Maharashtra, India \\ ${ }^{2}$ Department of Mathematics, Pratisthan Mahavidyalaya Paithan, Maharashtra, India \\ ${ }^{3}$ Department of Mathematics, Dr.Babasaheb Ambedkar Marathwada University, Aurangabad, Maharashtra, India
}

\begin{abstract}
This study analyzes the influence of internal heat generation on the temperature distribution, displacement and thermal stresses in a thick circular plate described as $0 \leq r \leq a,-h \leq z \leq h$ within a context of uncoupled thermoelasticity. A direct transient thermoelastic problem of circular plate because of internal heat generation with radiation kind of boundary conditions is discussed. The third kind boundary condition is kept on upper and lower plane surface of the plate. Over outer curved surface, $(r=a)$, temperature is kept at $f(z, t)$. The controlling heat conduction equation was completed through finite Hankel and Marchi Fasulo integral transform. The outcomes were acquired in way of Bessel's function in the form of infinite series. Mathematical technique is employed for aluminium (pure) material plate and the numerical solutions were discussed and represented graphically.

KEYWORDS: Thermal Stresses, Thick Circular Plate, Internal Heat Generation
\end{abstract}

Received: Dec 19, 2019; Accepted: Jan 11, 2020; Published: Apr 10, 2020; Paper Id.: IJMPERDAPR2020124

\section{INTRODUCTION}

Thermoelasticity deals with the effect of the thermal condition of a solid due to application of stresses and using the reverse effect, which of deformation. Thermoelasticity has been aroused by the variety of technology sciences. Modern advancements of nuclear engineering, aeronautics and astronautics, as well as a remarkable advancement in the subject of aircraft and machine formation have lead to many challenges where thermal stresses acts as a part of major significance. Unexpected and ongoing improvement of attentiveness within this subject grow throughout the next half of the twentieth century, where non-isothermal issues of the concept of elasticity turns into highly significant.

To begin with, in this area of aeronautics, the large velocity of contemporary aircrafts contribute to a aerodynamic heating, where create extreme thermal stresses, decreasing the potency of aircrafts formation. Next, in the nuclear area, the very substantial temperature also its gradients emerging within nuclear reactor affect its design and operations.

From the engineering of jet and rocket motors, the maximum temperatures related to combustion methods would be the source of undesirable thermal stresses. Therefore, the several issues where the thermal stresses acts as a significant, at times the critical function, happen in steam and gas turbines, in aviation formations, chemical technology, in nuclear technology, in the science of space vehicles and missiles, as well as in ship building, in which, curiously enough ship fractures were usually credited to thermal stresses of average strengths. Number of studies were completed to forecast deformation as well as heat flow in a continuum within the area of 
thermoelasticity throughout the past couple of years. It still requires high focus because of its extensive applications in diverse disciplines. Thermal stresses also act as a very important part within the area of chemical technology, at the technologies of space vehicles and missiles. The thermoelastic behaviour of solids symbolizing the bases of containers for hot gases or liquids, at the bases for furnaces, in applications including turbine engines, flywheels, gears etc., is highly significant.

Nowacki [8] studied thermal stresses in circular plate exposed to a axisymmetric temperature distribution over the top face without temperature on the bottom face and the circular edge. Marchi, E. and Fasulo, A. [5] researched heat conduction in business of hollow cylinder with radiation. Roy Choudhary [11] talked about the Quasi-static thermal deflection of a clamped circular plate because of ramp-type heating. Qian and Batra [10] analyzed transient thermoelastic problem of thick functionally graded plate. Gogulwar and Deshmukh [3] established thermal stresses in thin circular plate using heat sources. Additionally Nasser M.EI-Maghray [6] solved two-dimensional issue of thick plate with heat resources in generalized thermoelasticity. Deshmukh K. C. et.al. [1] examined quasi static thermal deflection of a thin clamped circular plate because of heat generation. Gaikwad and Ghadle [2] analyzed non-homogeneous heat conduction issue and its thermal deflection as a result of internal heat sources within a thin hollow circular disk. Khan, Khalsa and Varghese [4] explored reverse quasi static unsteady state thermal stresses within a thick circular plate.

The research deals with transient thermoelastic issue of a thick circular plate covering the gap $D: 0 \leq r \leq a,-h \leq z \leq h$, because of heat generation with radiation boundary conditions. This finite Hankel transform and finite Marchi Fasulo transform method was utilized to locate the solution of the issue. The outcomes existed here to be more helpful in technology issues especially, at the conclusion of the condition of stresses in circular plate constituting bases of containers for hot gases or liquids, in the bases for furnaces, etc.

\section{THE TRANSFORMATION AND ITS ESSENTIAL PROPERTY}

2.1 The finite Hankel transform over the variable $r$ and its inversion described as in Özişik[9] is

$$
\begin{aligned}
& \bar{T}\left(\xi_{m}, z, t\right)=\int_{r^{\prime}=0}^{a} r^{\prime} K_{0}\left(\xi_{m}, r^{\prime}\right) T\left(r^{\prime}, z, t\right) d r^{\prime} \\
& T(r, z, t)=\sum_{m=1}^{\infty} \quad K_{0}\left(\xi_{m}, r\right) \bar{T}\left(\xi_{m}, z, t\right)
\end{aligned}
$$

where,

$K_{0}\left(\xi_{m}, r\right)=\frac{\sqrt{2}}{a} \frac{J_{0}\left(\xi_{m} r\right)}{J_{0}{ }^{\prime}\left(\xi_{m} a\right)}$

and the eigen value $\xi_{m}$ are the positive roots of the transcendental equation

$J_{0}(\xi a)=0$

where $J_{n}(x)$ is Bessel function of the first kind of order $n$.

The finite Hankel transform $H$ defined in equation (2.1.1) satisfies the relation

$H\left[\frac{\partial^{2} T}{\partial r^{2}}+\frac{1}{r} \frac{\partial T}{\partial r}\right]=-\xi_{m}^{2} \bar{T}\left(\xi_{m}, z, t\right)$ 
2.2 The finite Marchi Fasulo integral transform of $f(z),-h<z<h$ was described in [3] as

$$
\bar{F}(n)=\int_{-h}^{h} f(z) P_{n}(z) d z
$$

Then

$$
f(z)=\sum_{n=1}^{\infty} \frac{\bar{F}(n)}{\lambda_{n}} P_{n}(z)
$$

where,

$$
\begin{aligned}
& P_{n}(z)=Q_{n} \cos \left(a_{n} z\right)-W_{n} \sin \left(a_{n} z\right) \\
& Q_{n}=a_{n}\left(\alpha_{1}+\alpha_{2}\right) \cos \left(a_{n} h\right)+\left(\beta_{1}-\beta_{2}\right) \sin \left(a_{n} h\right) \\
& W_{n}=\left(\beta_{1}+\beta_{2}\right) \cos \left(a_{n} h\right)+\left(\alpha_{2}-\alpha_{1}\right) a_{n} \sin \left(a_{n} h\right) \\
& \lambda_{n}=\int_{-h}^{h} P_{n}^{2}(z) d z=h\left[Q_{n}{ }^{2}+W_{n}{ }^{2}\right]+\frac{\sin \left(2 \mathrm{a}_{n} \mathrm{~h}\right)}{2 \mathrm{a}_{\mathrm{n}}}\left[Q_{n}{ }^{2}-W_{n}{ }^{2}\right]
\end{aligned}
$$

The eigen values $a_{n}$ satisy the equation

$$
\begin{aligned}
{\left[\alpha_{1} a \cos (a h)\right.} & \left.+\beta_{1} \sin (a h)\right] \times\left[\beta_{2} \cos (a h)+\alpha_{2} a \sin (a h)\right] \\
& =\left[\alpha_{2} a \cos (a h)-\beta_{2} \sin (a h)\right] \times\left[\beta_{1} \cos (a h)-\alpha_{1} a \sin (a h)\right]
\end{aligned}
$$

$\alpha_{1}, \alpha_{2}, \beta_{1}$ and $\beta_{2}$ are constants.

The sum in (2.2.2) must be taken on $n$ corresponding to the positive roots of the equation (2.2.4)

The transform (2.2.1) has the following property:

$$
\begin{aligned}
\int_{-h}^{h} \frac{\partial^{2} f(z)}{\partial z^{2}} & P_{n}(z) d z \\
& =\frac{P_{n}(h)}{\alpha_{1}}\left[\beta_{1} f(z)+\alpha_{1} \frac{\partial f(z)}{\partial z}\right]_{z=h}-\frac{P_{n}(-h)}{\alpha_{2}}\left[\beta_{2} f(z)+\alpha_{2} \frac{\partial f(z)}{\partial z}\right]_{z=-h}-a_{n}{ }^{2} \bar{F}(n)
\end{aligned}
$$

\section{STATEMENT OF THE PROBLEM}

Consider thick circular plate of radius $a$ and thickness $2 h$ covering those gaps

$D: 0 \leq r \leq a,-h \leq z \leq h$, the material is homogenous and isotropic. Initially, the circular plate is at zero temperature. On upper plane surface $(z=h)$, the third kind boundary condition was kept at $g_{1}(r, t)$, whereas on lower plane surface $(z=-h)$, the third kind boundary condition was kept at $g_{2}(r, t)$, where $g_{1}(r, t)$ and $g_{2}(r, t)$ are known functions of $r$ and $t$. On outer curved surface $(r=a)$, temperature is maintained at $f(z, t)$. For time $t>0$, heat is generated in the plate at the rate $Q(r, z, t)$. The differential equation governing the displacement potential function $\Phi(r, z, t)$ as in Nowacki[8] is

$$
\nabla^{2} \Phi=K T
$$

where, the Laplacian operator $\nabla^{2}$ is

$$
\nabla^{2}=\frac{\partial^{2}}{\partial r^{2}}+\frac{1}{r} \frac{\partial}{\partial r}+\frac{\partial^{2}}{\partial z^{2}}
$$


and

$$
K=\left(\frac{1+v}{1-v}\right) \alpha
$$

where $K, v$ and $\alpha$ are restraint coefficient, Poisson's ratio along with linear coefficient of thermal expansion of the material of the plate respectively. $\Phi$ is Goodier's thermoelastic displacement potential and $T$ is the temperature distribution of the plate fulfilling the differential equation as Noda [7] is

$$
\nabla^{2} T+\frac{Q(r, z, t)}{\lambda}=\frac{1}{k} \frac{\partial T}{\partial t}
$$

Subject to initial condition

$$
\left.T(r, z, t)\right|_{t=0}=0, \quad 0 \leq r \leq a,-h \leq z \leq h
$$

The boundary conditions are

$$
\begin{array}{lc}
\left.T\right|_{r=a}=f(z, t), & -h \leq z \leq h, t>0 \\
h_{1} T+\left.\frac{\partial T}{\partial z}\right|_{z=h}=g_{1}(r, t), & 0 \leq r \leq a, t>0 \\
h_{2} T+\left.\frac{\partial T}{\partial z}\right|_{z=-h}=g_{2}(r, t), & 0 \leq r \leq a, t>0
\end{array}
$$

where $k$ is thermal diffusivity of material of the plate, $\lambda$ is thermal conductivity of material of the plate,

$Q$ is the internal heat also $h_{1}, h_{2}$ are heat transfer coefficients.

The displacements in terms of $\Phi$ and $M$ as in Noda [7] are

$u_{r}=\frac{\partial \Phi}{\partial r}-\frac{\partial^{2} M}{\partial r \partial z}$

$u_{z}=\frac{\partial \Phi}{\partial z}+2(1-v) \nabla^{2} M-\frac{\partial^{2} M}{\partial z^{2}}$

$M$ must satisfy the equation

$\nabla^{2} \nabla^{2} M=0$

The components of stress as in Noda [7] are

$$
\begin{aligned}
& \sigma_{r r}=2 G\left\{\left(\frac{\partial^{2} \Phi}{\partial r^{2}}-K T\right)+\frac{\partial}{\partial z}\left(v \nabla^{2} \mathrm{M}-\frac{\partial^{2} \mathrm{M}}{\partial \mathrm{r}^{2}}\right)\right\} \\
& \sigma_{\theta \theta}=2 G\left\{\left(\frac{1}{r} \frac{\partial \Phi}{\partial r}-K T\right)+\frac{\partial}{\partial z}\left(v \nabla^{2} \mathrm{M}-\frac{1}{r} \frac{\partial \mathrm{M}}{\partial r}\right)\right\} \\
& \sigma_{z z}=2 G\left\{\left(\frac{\partial^{2} \Phi}{\partial z^{2}}-K T\right)+\frac{\partial}{\partial z}\left((2-v) \nabla^{2} \mathrm{M}-\frac{\partial^{2} \mathrm{M}}{\partial \mathrm{z}^{2}}\right)\right\} \\
& \sigma_{r z}=2 G\left\{\frac{\partial^{2} \Phi}{\partial r \partial z}+\frac{\partial}{\partial r}\left((1-v) \nabla^{2} \mathrm{M}-\frac{\partial^{2} \mathrm{M}}{\partial \mathrm{z}^{2}}\right)\right\}
\end{aligned}
$$

where $G$ is shear modulus.

For traction free surface, stress function 
$\sigma_{\theta z}=\sigma_{r \theta}=0$ at $z= \pm h$ for thick plate.

Equations (3.1) to (3.13) constitute the mathematical formulation of the boundary value problem of thermoelasticity under thought.

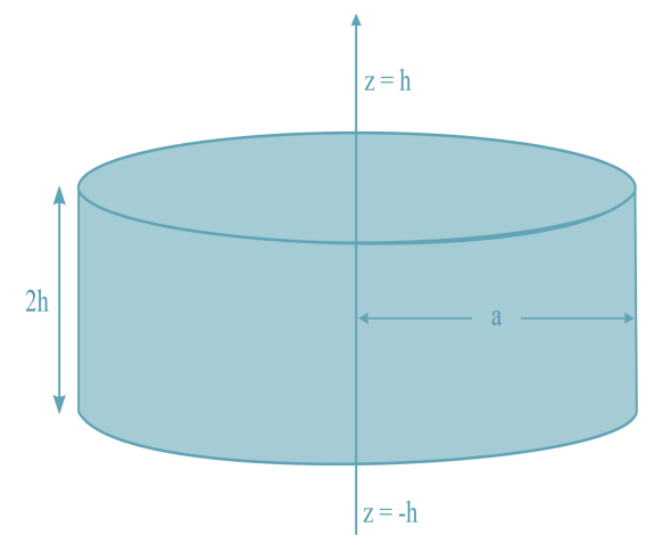

Figure 1: The Geometry of the Problem.

\section{SOLUTION OF THE PROBLEM}

Applying rule described in above [2.1] to the equation (3.2), and using (2.1.5), we have,

$-\xi_{m}^{2} \bar{T}\left(\xi_{m,}, t\right)+\frac{d^{2} \bar{T}}{d z^{2}}\left(\xi_{m}, z, t\right)+\frac{\bar{Q}\left(\xi_{m}, z, t\right)}{\lambda}=\frac{1}{k} \frac{d \bar{T}}{d t}$

where $\bar{T}$ denotes the finite Hankel transform of $\mathrm{T}$ and $\xi_{m}$ is the Hankel transform parameter.

Additionally, applying rule described in [2.2] to above equation (4.1),

$\frac{d \bar{T}^{*}}{d t}+k q^{2} \bar{T}^{*}=r$

where,

$$
q^{2}=\xi_{m}^{2}+a_{n}^{2}
$$

and

$$
r=k\left\{P_{n}(h) \bar{g}_{1}-P_{n}(-h) \bar{g}_{2}+\frac{\bar{Q}^{*}\left(\xi_{m}, n, t\right)}{\lambda}\right\}
$$

where, $T^{*}$ denotes the Marchi-Fasulo transform of $\mathrm{T}$ and $n$ is the Marchi-Fasulo transform parameter.

Solving equation (4.2), which is a $1^{\text {st }}$ order differential equation, we have,

$\bar{T}^{*}\left(\xi_{m}, n, t\right)=e^{-k q^{2} t}\left\{\int_{0}^{t} \gamma e^{k q^{2} t^{\prime}} d t^{\prime}+C\right\}$

From (3.3) and (4.3), C = 0.

Hence,

$\bar{T}^{*}\left(\xi_{m}, n, t\right)=e^{-k q^{2} t}\left\{\int_{0}^{t} \gamma e^{k q^{2} t^{\prime}} d t^{\prime}\right\}$

Applying rule in (2.2.2) to the equation (4.4), we get, 
$\bar{T}\left(\xi_{m}, z, t\right)=\sum_{n=1}^{\infty} \frac{P_{n}(z)}{\lambda_{n}} e^{-k q^{2} t} \int_{0}^{t} \gamma e^{k q^{2} t^{\prime}} d t^{\prime}$

Again, applying rule in (2.1.2) to the equation (4.5), we get

$T(r, z, t)=\frac{\sqrt{2}}{a} \sum_{m} \sum_{n} \frac{J_{0} \quad\left(\xi_{m} r\right)}{J_{0}{ }^{\prime}\left(\xi_{m} a\right)} \frac{P_{n} \quad(z)}{\lambda_{n}} \Psi$

where,

$$
\Psi=e^{-k q^{2} t}\left\{\int_{0}^{t} \gamma e^{k q^{2} t^{\prime}} d t^{\prime}\right\}
$$

and the eigen values $\xi_{m}$ are the positive roots of the transcendental equation $J_{0}(\xi a)=0$.

The expression in (4.6) gives the temperature distribution.

Let us consider Michell's function $M$, fulfilling condition (3.9) as

$M(r, z)=\frac{\sqrt{2}}{a} \sum_{m} \sum_{n} \frac{J_{0} \quad\left(\xi_{m} r\right)}{J_{0}{ }^{\prime}\left(\xi_{m} a\right)} \frac{P_{n}(z)}{\lambda_{n}}$

Using (3.1) and (4.6), we get displacement potential $\Phi$ as

$\Phi=\frac{\sqrt{2}}{a} R \sum_{m} \sum_{n} \frac{J_{0}\left(\xi_{m} r\right)}{J_{0}{ }^{\prime}\left(\xi_{m} a\right)} \frac{P_{n}(z)}{\lambda_{n}} S(t)$

where

$R=k\left(\frac{1+v}{1-v}\right) \alpha$, and $S(t)=\int \Psi d t$

\section{DETERMINATION OF DISPLACEMENT FUNCTION}

Substituting equations (4.7) and (4.8) in equation (3.7) and (3.8), we get

$$
\begin{aligned}
& u_{r}=\frac{\sqrt{2}}{a} \sum_{m} \sum_{n} \frac{\xi_{m} J_{0}{ }^{\prime}\left(\xi_{m} r\right)}{J_{0}{ }^{\prime}\left(\xi_{m} a\right)}\left[R \frac{P_{n}(z)}{\lambda_{n}} S(t)-\frac{P_{n}{ }^{\prime}(z)}{\lambda_{n}}\right] \\
& u_{z}=\frac{\sqrt{2}}{a}\left\{\begin{array}{c}
\sum_{m} \sum_{n} R \frac{J_{0}\left(\xi_{m} r\right)}{J_{0}{ }^{\prime}\left(\xi_{m} a\right)} \frac{P_{n}{ }^{\prime}(z)}{\lambda_{n}} S(t)-\sum_{m} \sum_{n} \frac{J_{0}\left(\xi_{m} r\right)}{J_{0}{ }^{\prime}\left(\xi_{m} a\right)} \frac{P_{n}{ }^{\prime \prime}(z)}{\lambda_{n}} \\
\left.+2(1-v)\left[\begin{array}{c}
\sum_{m} \sum_{n}\left(\xi_{m}{ }^{2} \frac{J_{0}^{\prime \prime}\left(\xi_{m} r\right)}{J_{0}{ }^{\prime}\left(\xi_{m} a\right)}+\frac{1}{r} \frac{\xi_{m} J_{0}{ }^{\prime}\left(\xi_{m} r\right)}{J_{0}{ }^{\prime}\left(\xi_{m} a\right)}\right) \frac{P_{n}(z)}{\lambda_{n}} \\
+\sum_{m} \sum_{n} \frac{J_{0}\left(\xi_{m} r\right)}{J_{0}{ }^{\prime}\left(\xi_{m} a\right)} \frac{P_{n}{ }^{\prime \prime}(z)}{\lambda_{n}}
\end{array}\right]\right\}
\end{array}\right.
\end{aligned}
$$

Substituting equations (4.7) and (4.8) in equations (3.10) to (3.13), we obtain

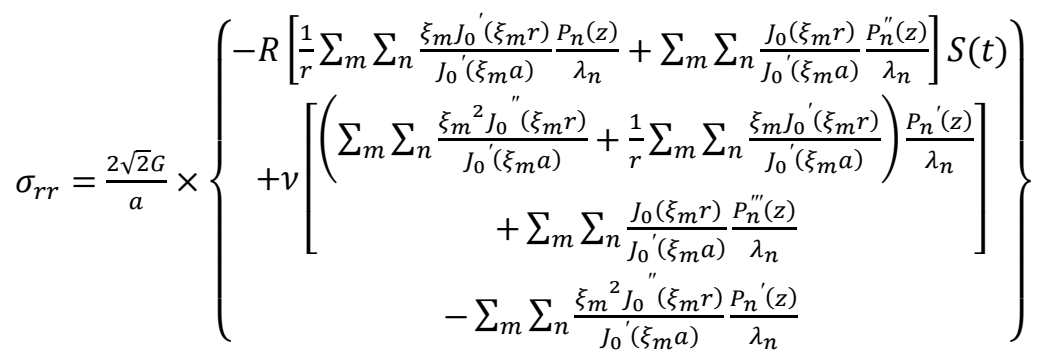




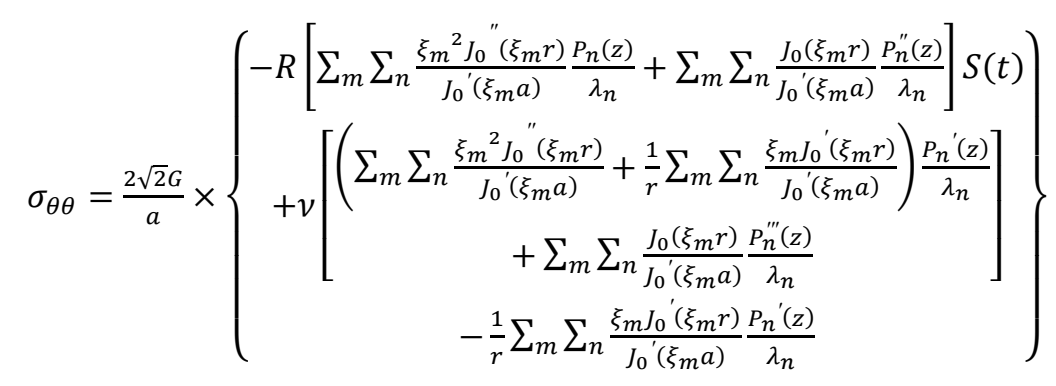

$$
\begin{aligned}
& \sigma_{z z}=\frac{2 \sqrt{2} G}{a} \times\left\{\begin{array}{c}
-R\left[\sum_{m} \sum_{n} \frac{\xi_{m}{ }^{2} J_{0}{ }^{\prime \prime}\left(\xi_{m} r\right)}{J_{0}{ }^{\prime}\left(\xi_{m} a\right)} \frac{P_{n}(z)}{\lambda_{n}}+\frac{1}{r} \sum_{m} \sum_{n} \frac{\xi_{m} J_{0}{ }^{\prime}\left(\xi_{m} r\right)}{J_{0}{ }^{\prime}\left(\xi_{m} a\right)} \frac{P_{n}(z)}{\lambda_{n}}\right] S(t) \\
+(2-v)\left[\left(\sum_{m} \sum_{n} \frac{\xi_{m}{ }^{2} J^{\prime \prime}\left(\xi_{m} r\right)}{J_{0}{ }^{\prime}\left(\xi_{m} a\right)}+\frac{1}{r} \sum_{m} \sum_{n} \frac{\xi_{m} J_{0}{ }^{\prime}\left(\xi_{m} r\right)}{J_{0}{ }^{\prime}\left(\xi_{m} a\right)}\right) \frac{P_{n}{ }^{\prime}(z)}{\lambda_{n}}\right] \\
+\sum_{m} \sum_{n} \frac{J_{0}\left(\xi_{m} r\right)}{J_{0}{ }^{\prime}\left(\xi_{m} a\right)} \frac{P_{n}^{\prime \prime \prime}(z)}{\lambda_{n}} \\
-\sum_{m} \sum_{n} \frac{J_{0}\left(\xi_{m} r\right)}{J_{0}{ }^{\prime}\left(\xi_{m} a\right)} \frac{P_{n}{ }^{\prime \prime}(z)}{\lambda_{n}}
\end{array}\right\}
\end{aligned}
$$

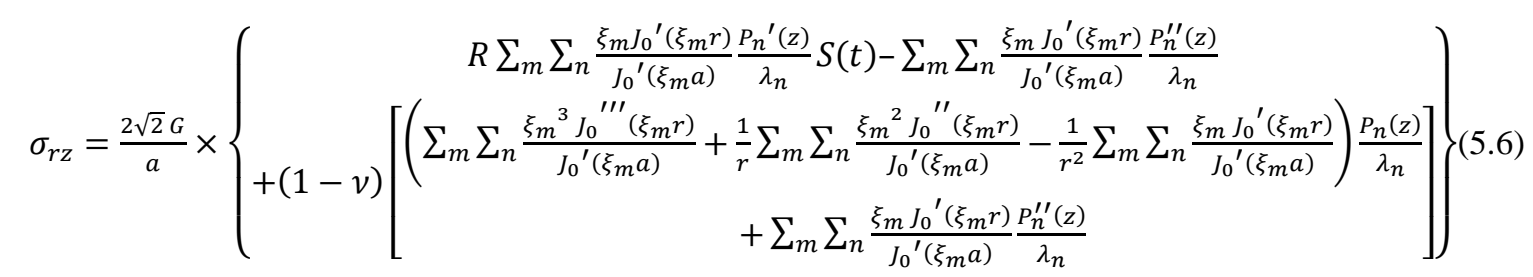

\section{NUMERICAL RESULTS AND DISCUSSIONS}

$$
\begin{aligned}
& \text { Set } f(z, t)=A\left(1-e^{-t}\right) z, \\
& \begin{array}{l}
g_{1}(r, t)=g_{2}(r, t)=B\left(1-e^{-t}\right) r \\
Q(r, z, t)=\delta\left(r-r_{0}\right) \delta\left(z-z_{0}\right) \delta\left(t-t_{0}\right)
\end{array}
\end{aligned}
$$

where $\delta$ is the Dirac-delta function.

The numerical problem has been completed for aluminum (pure) plate with parameters $\mathrm{a}=2 \mathrm{~m}, \mathrm{~h}=1 \mathrm{~m}, \mathrm{t}=1 \mathrm{hr}$.

Thermal diffusivity $k=84.18 \times 10^{-6} \mathrm{~m}^{2} \mathrm{~s}^{-1}$

Thermal conductivity $\lambda=204.2 \mathrm{~W} / \mathrm{mk}$

Density $\rho=2707 \mathrm{~kg} / \mathrm{m}^{3}$

Specific heat $C_{\rho}=896 \mathrm{~J} / \mathrm{kgK}$

Poisson ratio $v=0.35$

Coefficient of linear thermal expansion $\alpha=22.2 \times 10^{-6} / \mathrm{K}$

Lame constant $\mu=26.67$

Also, $\xi_{1}=1.2024, \xi_{2}=2.7601, \xi_{3}=4.3269, \xi_{4}=5.8958, \xi_{5}=7.4655, \xi_{6}=9.0356$ are the positive roots of

the transcendental equation $\boldsymbol{J}_{\mathbf{0}}(\mathbf{2} \xi)=\mathbf{0}$ as in [9] 


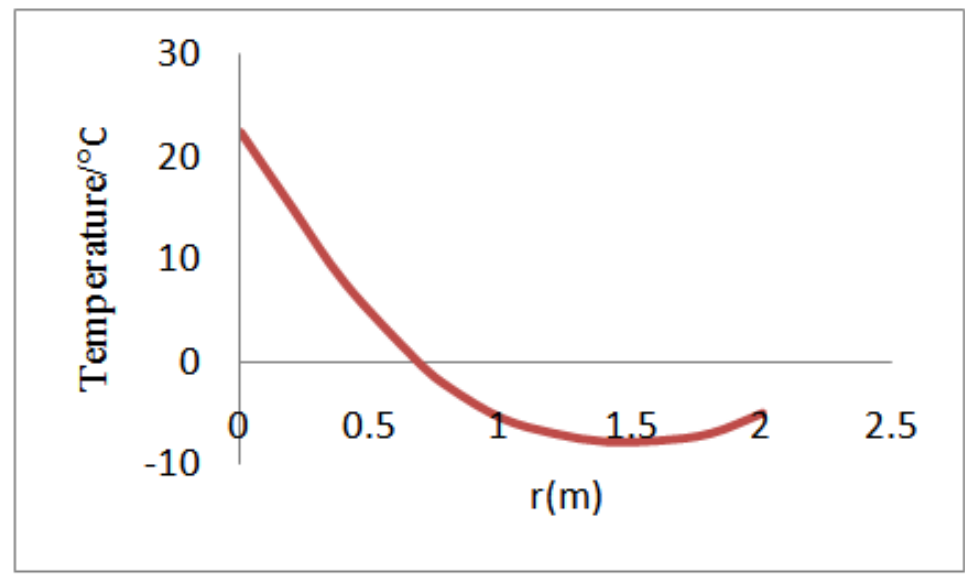

Figure 2: Temperature Distribution.

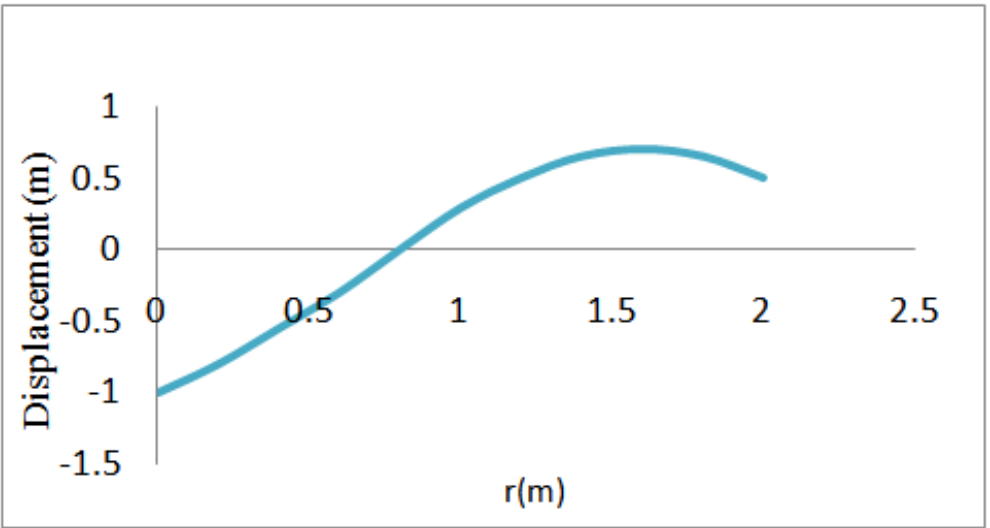

Figure 3: Displacement Function.



Figure 4: Radial Stress Function. 


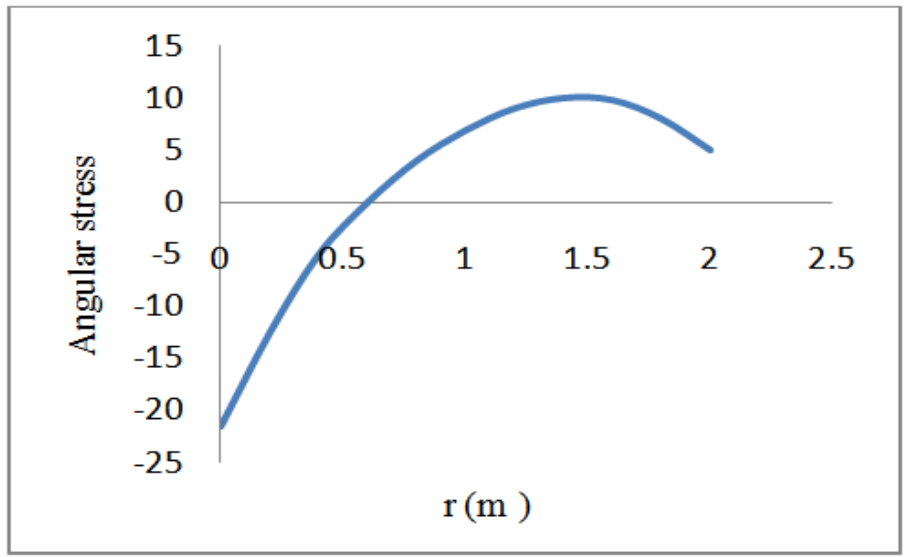

Figure 5: Angular Stress Function.

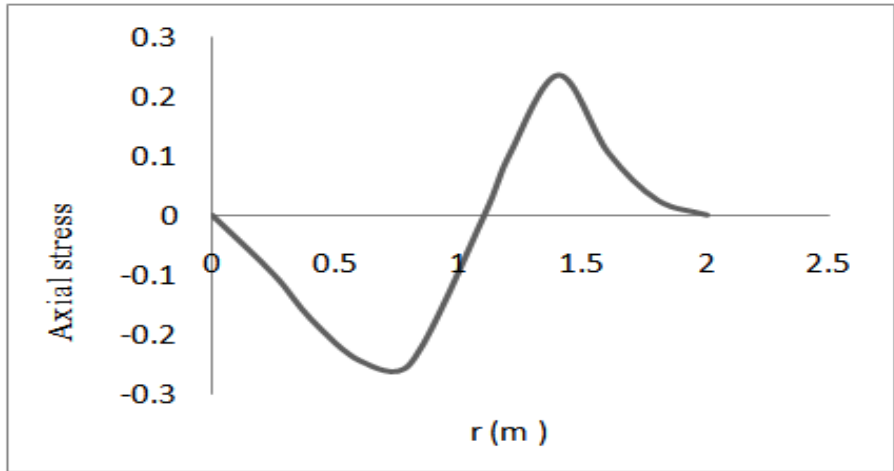

Figure 6: Axial Stress Function.

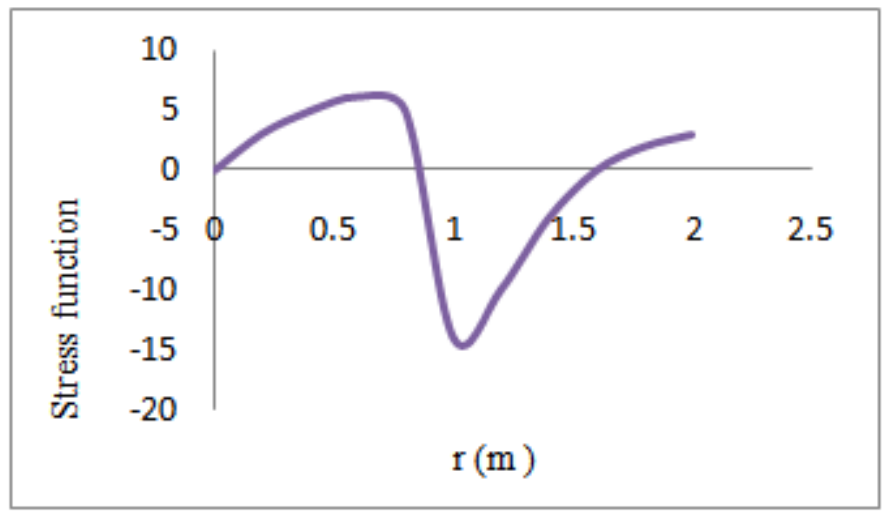

Figure 7: Stress Function.

From figure 2, it is found that, temperature turns maximum at the centre and reduces to the external circular edge.

As time passes, the temperature of plate rises because of internal heat source.

From figure3, the displacement increases to the external circular edge.

From figure 4, the radial stress turns nothing at the external circular boundary of plate.

The radial stress is compressive.

From figure 5, the angular stress go on increasing from the central part to the external circular edge. 
It is zero at $r=0.6$. The angular stress evolves as compressive stresses near the center and tensile stresses near the external circular edge of the plate.

From figure 6, the axial stress varies nonuniformly with radial direction and evolves compressive stresses in radial direction.

From figure 7, stress varies with radial direction because of effect of internal heat source. It takes value zero at the center.

\section{CONCLUSIONS}

From the research paper, the temperature distribution, displacement and thermal stresses of a thick circular plate were analyzed with identified boundary conditions. The significant role played by internal heat generation has been represented by Finite integral transform methods. The outcomes were acquired in terms of Bessel's function in the form of series. Because of internal heat, the radial stress develops as compressive stresses and the angular stresses evolves as compressive stresses near the center and tensile stresses near the external circular edge of the plate. The rate of deflection is proportional to time and increases as time passes. Substantial enhancement in the temperature occurs due to internal heat.

Any specific scenarios of particular interest can be defined through allocating correct values to the parameters and functions in expressions. The outcomes which were acquired can be helpful to the outline of structure or machines in engineering applications.

\section{REFERENCES}

1. Deshmukh K. C., Warbhe S. D.And Kulkarni V. S., 2009, Quasi Static Thermal Deflection Of A Thin Clamped Circular Plate Due To Heat Generation, Journal Of ThermalStresses, 32(9), pp. 877-886.

2. Gaikwad K.R. And Ghadle K. P., 2012, Nonhomogeneous Heat Conduction Problem And Its Thermal Deflection Due To Internal Heat Generation In A Thin Hollow Circular Disk,Journal Of Thermal Stresses, 35: No. 6,pp. 485-498.

3. Gogulwar, V. S.And Deshmukh, K. C., 2005, Thermal Stresses In A Thin Circular Plate With Heat Sources, Journal Of Indian Academy Of Mathematics, Vol.27, No. 1.

4. Khan Ishaque, KhalsaLalsinghAndVarghese Vinod, 2017, Inverse Quasi Static Unsteady State Thermal Stresses In A Thick Circular Plate, Cogent Mathematics, 4(1), pp. 1-10.

5. Marchi, E. And Fasulo, A. (1967) :Heat Conduction In Sector Of Hollow Cylinder With Radiation,Atti, Della Acc. Sci. Di. Torino, 1, pp. 373-382.

6. Nasser M.EI-Maghraby, 2005, "Two Dimensional Problem For A Thick Plate With Heat Sources In Generalized Thermoelasticity", Journal Of Thermal Stresses, Vol. 28, pp. 1227-1241.

7. Noda, N.; Hetnarski, R. B. And Tanigawa, Y., 2003, "Thermal Stresses", Taylor AndFrancis, New York, 2nd Ed., pp. 259-261.

8. Nowacki, W., 1957, "The State Of Stresses In A Thick Circular Plate Due To Temperature Field", Bull. Acad. Polon. Sci., Scr. Scl. Tech., Vol.5, pp.227.

9. Özişik, M.N.(1968):Boundary Value Problems Of Heat Conduction, InternationalTextbookCompany, Scranton, Pennsylvania, pp.9-181. 
10. Qian, L. F., And Batra, R. C., 2004, Transient Thermoelastic Deformation Of A Thick Functionally Graded Plate, Journal OfThermal Stresses, Vol. 27, pp.705-740.

11. Roy Choudhary, S.K., 1973, A Note On Quasi-Static Thermal Deflection Of A Thin Clamped Circular Plate Due To RampType Heating Of A Concentric Circular Region Of The Upper Face, Journal Of The Franklin Institute, Vol.206, No 3, Sept., pp.. 213-219.

12. Yogita M. Ahire, Ahmed A. Hamoud\&Kirtiwant P. Ghadle, "Analysis of Thermal Stresses in Thin Circular Plate Due to Moving Heat Source", IJMPERD, Vol. 9, Issue 3, pp. 1285-1292

13. Kamini K. Chaudhari\&Chandrashekhar S. Sutar, "Thermoelastic Modelling of Rectangular Plate under the Hyperbolic Heat Conduction with an Internal Heat Source”, IJMPERD, Vol. 9, Issue 6, pp. 859-872 

